\title{
Light Quark Spectroscopy and Charm Decays
}

\author{
Alberto Reis \\ Centro Brasileiro de Pesquisas Físicas, Rua Dr. Xavier Sigaud, 150, 22290-970, Rio de Janeiro, Brazil
}

Received on 14 August, 2003.

\begin{abstract}
The connection between light quark spectroscopy and hadronic decays of D mesons is discussed, with emphasis on the physics of the light scalar mesons. Recent results from charm decays are presented.
\end{abstract}

\section{Introduction}

Forty years have passed since the birth of the Constituent Quark Model (CQM). This model provided a very successful description of almost all the hadronic spectrum. The nonets of pseudo-scalar, vector and tensor mesons are now well identified. There is, however, one remaining and crucial problem: the identification of the scalar meson nonet(s). The solution of this enigma is of vital importance for understanding QCD at the low energy limit.

On the other side, there has been tremendous progress in charm physics in the past decade. High quality data allowed the basic properties of charm mesons to be well measured. Recently hadronic decays of charm mesons started being used to study properties of scalar mesons, abundant products of these decays.

Charm decays have unique features, making them a very interesting tool for light quark spectroscopy: large couplings to scalar mesons and very small (less than 10\%) nonresonant components; an initial state which is always well defined: the spin-0 D meson; and a spectrum that is not constrained by isospin and parity conservation.

There are, however, some conceptual issues related to the formalism commonly used in the analysis of resonant substructure of hadronic decays: the correct representation of overlapping broad states, which is closely connected to the issue of formulating the unitarity constraint in three and four-body problems. Moreover, there is the question of how to relate the observations from charm to those from scattering.

In what follows I will briefly state the problem of the scalar mesons. Then I will discuss how we can use charm decays for new insights on the scalars. Finally, I will discuss the picture so far offered by hadronic decays of charm.

\section{The puzzling light scalars}

The light scalars are, in some sense, victims of their own simplicity. Due to their broad widths and the lack of a distinctive angular distribution, the distinction between scalar mesons and the non-resonant background is rather difficult. Moreover, there are many overlapping states within a limited range of the mass spectrum (up to $1.8 \mathrm{GeV}$ ). An additional difficulty is the fact that non- $q \bar{q}$ states, like the lightest scalar glueball or multiquark states, all sharing the same quantum numbers $\left(J^{P}=0^{+}\right)$, are expected to populate the same region of the spectrum. We can say that the identification of the scalar mesons will always be a difficult subject. Comprehensive reviews on scalar mesons can be found in [1] and references therein.

The main candidates, according to their isospin, are: $\mathrm{f}_{0}(600)$ or $\sigma(500), \mathrm{f}_{0}(980), \mathrm{f}_{0}(1370), \mathrm{f}_{0}(1500)$ and $\mathrm{f}_{0}(1710)$ $(I=0) ; \kappa(800)$ and $K_{0}^{*}(1430)(I=1 / 2) ; \mathrm{a}_{0}(980)$ and $\mathrm{a}_{0}(1450)(I=1)$.

If all these states are confirmed, we have 19 states! Too many candidates to fit even in two nonets. While the actual existence of some of these states - the $\sigma(500)$ and $\kappa(800)$ is controversial, other states just have poorly known parameters - $f_{0}(980), a_{0}(980), f_{0}(1370)$. The interpretation of most of the scalar candidates is also controversial. Are they genuine $q \bar{q}$ mesons or more complex objects? Take the case of the $\mathrm{a}_{0}(980)$, for instance. Its expected width is $500 \mathrm{MeV}$, according to the CQM, whereas the measured width is in the range 50-100 MeV. This fact leads to the interpretation of this state as a $q q \bar{q} \bar{q}$.

The most problematic states are the isoscalars. In addition to the controverse about the $\sigma(500)$, the nature of the $\mathrm{f}_{0}(980)$, there is the issue of the $\mathrm{f}_{0}$ family above $1 \mathrm{GeV}$ and a possible mixing with the scalar glueball [5]. The remaining of this note is devoted to isoscalars and to what can we learn about them from charm decays. The $I=1 / 2$ states states including the $\kappa(800)$, will be addressed in the talk by Carla Göbel, to appear in these proceedings.

\section{Charm decays and light scalars}

Hadronic decays of charm mesons are a natural place to look for scalars, with unique features that provide new and complementary insights on this problem.

Scalars are copiously produced in charm decays. In 3 and 4-body hadronic decays of D mesons, one always has a $\pi \pi$, a $K \pi$ or a $K K$ pair, important decay modes of scalar mesons. The quantum interference between broad scalars and the usually large non-resonant background, which is a 
plague in scattering experiments, does not affect charm decays because the non-resonant component is always very small.

But the most appealing features of D decays, when compared to scattering experiments, are related to the difference in the constraints that build the $\pi \pi, K \pi$ and $K K$ spectra. In scattering experiments, only the strong interaction is involved. The observed spectrum is determined by the conservation of isospin and parity. Parity and isospin are violated in $\mathrm{D}$ meson decays, where the observed spectrum is determined by the quark content of the initial state, after the weak decay of the c quark.

It is illustrative to compare, for instance, in the $\pi^{+} \pi^{-} \pi^{+}$ final state, the Dalitz plots from $D^{+}, D_{s}^{+}$decays[2, 3] and from $p \bar{n}$ annihilation[4], which is most similar to $\mathrm{D}$ decays. The differences due to production dynamics are apparent at a glance. Comparing the $D^{+}$and $D_{s}^{+}$Dalitz plots we see clearly the effects of the different quark content of the initial state.

There is a related aspect which is also crucial: the bulk of the hadronic decay widths can be explained by a model in which resonances couple directly to the D meson. There is no need to add couplings to other states, like glueballs: $q \bar{q}$ states alone seem to be enough to account for the observed rates. Take the decay $D_{s}^{+} \rightarrow K^{-} K^{+} \pi^{+}$as a typical case. The main amplitudes are the external and internal W-radiation. The decay modes corresponding to these amplitudes are $D_{s}^{+} \rightarrow \phi \pi^{+}$and $D_{s}^{+} \rightarrow K^{*} K^{-}$. These modes account for almost $100 \%$ of the $D_{s}^{+} \rightarrow K^{-} K^{+} \pi^{+}$ decay rate. The same argument could be made using many other final states. Hadronic D decays are an extremely complex process, and these types of quark diagrams are only an aproximation. This descripton, however, seems to work fairly well.

It is generally accepted that a resonance, being a real particle, must have the same parameters in whichever process it appears. The question one may ask is whether the states produced in different processes are really the same. Consider, for instance, the $f_{0}(1370), f_{0}(1500)$ and $f_{0}(1710)$ imbroglio. All three states have been observed by many experiments, with fairly well measaured parameters (except for the $f_{0}(1370)$ ). But according to the CQM, only two $q \bar{q}$ states are expected: one being mostly $s \bar{s}$ and another being mostly $u \bar{u}+d \bar{d}$. So, the three $f_{0}$ 's could not belong to the same $q \bar{q}$ multiplet.

Glueballs are expected to be produced in "gluon-rich" reactions, like central production, in addition to the genuine $q \bar{q}$ mesons. Mixing between the scalar glueball and the $q \bar{q}$ states is expected[5]. If this is really the case, then the observed states would be mixtures of $q \bar{q}$ and $g g$, rather than pure states.

On the other hand, in a "gluon-poor" reaction, like D decays, glueballs are not expected to be produced. In D decays one would access directly the $q \bar{q}$ states with no mixing. In this case masses and widths measured in charm decays would be different than those obtained in central production. Also, the number of states present in $\mathrm{D}$ decays would be smaller.

One last aspect deserves some attention: the role of fi- nal state interactions in charm decays. The Dalitz plots of charm decays can only be describe by models allowing interference between amplitudes in which the resonance and the bachelor pseudo-scalar are in different states of relative orbital angular momentum. The role the bachelor pseudoscalar plays is decisive, which seems not to be the case in $N \bar{N}$ annihilations. In this sense Dalitz plot and partial wave analysis are not quite the same. The case of the $D^{+} \rightarrow K^{-} \pi^{+} \pi^{+}[8]$ is typical. We see in the Dalitz plot that the upper lobe of the $K^{*}(892)$ band is shifted with respect to the lower one. This effect is caused by the interference between the $l=1 D^{+} \rightarrow K^{*}(892) \pi^{+}$and the $l=0$ amplitudes, like $D^{+} \rightarrow K_{0}^{*}(1430) \pi^{+}$.

We conclude this section by noting that relating results from scattering and charm decays is not so simple. The $D \rightarrow \pi \pi \pi$, for instance, cannot be explained on the basis of pure elastic $\pi \pi$ scattering. The energy dependent s-wave phase from $D \rightarrow \pi \pi \pi$ (or $K \pi \pi$ ) may not be the same as the $\pi \pi$ (or $K \pi$ ) phase shifts from peripheral hadron-hadron reactions.

\section{What have we learned so far from charm decays?}

There are only a few experimental results on light scalars from charm decays. I will concentrate on the isoscalars: the $\sigma$ and the $f_{0}$ family.

\section{1 $\sigma(\mathbf{5 0 0})$ or $f_{0}(\mathbf{6 0 0})$}

This is certainly the most controversial state. In charm decays it appears as an excess of signal events at low $\pi^{+} \pi^{-}$mass. This effect is observed in the Dalitz plots of $D^{+} \rightarrow \pi^{+} \pi^{-} \pi^{+}$, from E791[3] and FOCUS, and of $D^{0} \rightarrow \bar{K}^{0} \pi^{+} \pi^{-}$, from CLEO[6]. The same structure was also observed in $J / \psi \rightarrow \omega \pi^{+} \pi^{-}$decay, from BES[7]. No such effect is observed in $\pi^{+} \pi^{-}$scattering, where the $\sigma$ is interpreted not as a real particle, but as a dynamical threshold effect.

The best description of charm decay data requires the presence of a broad, scalar (in E791 analysis different spin assignments were also tested), complex amplitude at low $\pi^{+} \pi^{-}$mass. A crucial aspect is that good fits can only be obtained allowing the phase of this complex amplitude to vary across the Dalitz plot. The above experiments have fitted their data assuming a Breit-Wigner function for this state, although it is known that for states like the $\sigma$ a BreitWigner is only an approximation. Different functional forms may yield different values of mass and width. The very concept of mass and width is model dependent in this case. The CLEO Collaboration[6] do not claim evidence for the $\sigma$ meson due to the uncertainty in the best parameterization of this amplitude. Anyway, good fits were obtained in all cases, and the values for the mass and width (see table 1) are in good agreement $-M \sim 480 \mathrm{MeV}, \Gamma_{0} \sim 320 \mathrm{MeV}$. 
Table 1. Mass and width of the $\sigma$ assuming a Breit-Wigner model.

\begin{tabular}{|l|c|c|}
\hline experiment & mass $(\mathrm{MeV})$ & width $(\mathrm{MeV})$ \\
\hline \hline E791 & $478 \pm 29$ & $324 \pm 46$ \\
CLEO & $390 \pm 60$ & $282 \pm 77$ \\
BES & $513 \pm 32$ & $335 \pm 67$ \\
\hline
\end{tabular}

It would be interesting, definitely, to show the phase variation across the Dalitz plot without assuming any functional form for the $\sigma$ amplitude. This is, unfortunately, very difficult because it involves a very large number of free parameters. In any case, it remains to be explained why in charm decays the $\sigma$ seems to be a real particle, but not in low energy elastic $\pi^{+} \pi^{-}$scattering.

\section{$4.2 f_{0}(\mathbf{9 8 0})$}

The width of this state is poorly known. The reason is that the $f_{0}(980)$ seems to behave differently depending on the reaction in which it is produced. While in scattering it looks broader and with a large coupling to the $K \bar{K}$ channel, in charm decays it looks just like a narrow regular $q \bar{q}$ resonance decaying mostly into pions. In the decay $D_{s}^{+} \rightarrow \pi^{+} \pi^{-} \pi^{+}$ the $f_{0}(980) \pi^{+}$component correspond to over $50 \%$ of the decay rate.

E791 used a coupled channel Breit-Wigner (the Flatté formula) in its fit [2]. The coupling to $K \bar{K}$ channel was found to be consistent with zero. An equally good fit was obtained using a regular Breit-Wigner, yielding $\Gamma_{0}=(44 \pm 3)$ $\mathrm{MeV}$. This is in agreement with preliminary results from FOCUS $\left(\Gamma_{0} \sim 55 \mathrm{MeV}\right.$, from $\left.D_{s}^{+} \rightarrow \pi^{+} \pi^{-} \pi^{+}\right)$and BES $\left(\Gamma_{0} \sim 45 \mathrm{MeV}\right.$, from $J / \psi \rightarrow \phi \pi^{+} \pi^{-}$).

The large rate in $D_{s}^{+} \rightarrow \pi^{+} \pi^{-} \pi^{+}$suggests a strong affinity of the $f_{0}(980)$ with $s \bar{s}$, if we take the W-radiation amplitude to be the dominant decay mechanism. In spite of a large $s \bar{s}$ in its wave function, the lack of a significant coupling to $K \bar{K}$ is due essentially to the narrow $f_{0}(980)$ width.

The above situation reinforces the interpretation of this state as a 4-quark state surrounded by a $K \bar{K}$ molecular cloud. At short distances, as in D decays, we would access the $q q \bar{q} \bar{q}$ component, whereas in peripheral processes the molecular component would manifest itself.

\section{$4.3 f_{0}(\mathbf{1 3 7 0}) / f_{0}(\mathbf{1 5 0 0})$}

The situation here is still rather confusing. The third state of the $f_{0}$ family above $1 \mathrm{GeV}, f_{0}(1710)$, which would be mostly $s \bar{s}$, is difficult to access, since it lies near the edge of the $D_{s}^{+}$decay phase space.

Charm decays are useful not only to measure the $f_{0}(1370)$ and $f_{0}(1500)$ masses and widths, but also to infer the quark content of these two states. If both are $q \bar{q}$ resonances, both should appear in charm decays. If, in addition, there is a significant $s \bar{s}$ component in their wave function, these states should appear in the $D_{s}^{+} \rightarrow K^{+} K^{-} \pi^{+}$decay.

Both E791 and FOCUS/E687, when analysing the $D_{s}^{+} \rightarrow \pi^{+} \pi^{-} \pi^{+}$decay, have found that only one state is necessary to describe the Dalitz plot, although they do not agree on the measured parameters for this state. While E687 [10] found a state with mass near $1475 \mathrm{MeV}$ and a width of about $100 \mathrm{MeV}$ (very similar to FOCUS preliminary numbers, and very close to the well measured $f_{0}(1500)$ parameters), E791 [2] found a somewhat wider state with a lower mass: $M_{0}=(1434 \pm 20) \mathrm{MeV}, \Gamma_{0}=(172 \pm 32) \mathrm{MeV}$.

BaBar [9] have found no evidence of neither one of the $f_{0}$ states in the $D^{0} \rightarrow \bar{K}^{0} K^{+} K^{-}$decay. FOCUS have found a small component of $f_{0}(1370) \pi^{+}$in the $D_{s}^{+} \rightarrow$ $K^{+} K^{-} \pi$ decay, but the sum of all decay fractions is over $160 \%$. This is due to a large destructive interference, which is likely to be unphysical.

A large $D \rightarrow \pi^{+} \pi^{-} \pi^{+}$(and also $D \rightarrow \pi^{+} \pi^{0} \pi^{0}$ ) sample are necessary to disentangle the $f_{0}(1370) / f_{0}(1500)$ contribution. Apparently only one of the two $f_{0}$ would be a $q \bar{q}$ state (mostly $n \bar{n}$ ), reinforcing the interpretation of the other one as the ground-state scalar glueball.

\section{Conclusions}

The picture offered so far by charm decays points to the existence of two scalar meson nonets, one having states with mass below $1 \mathrm{GeV}$ and the other with masses above $1 \mathrm{GeV}$.

In the low mass states we have the large rates of the $\sigma \pi$ in $D^{+} \rightarrow \pi^{+} \pi^{-} \pi^{+}$and of the $f_{0}(980) \pi$ in $D_{s}^{+} \rightarrow \pi^{+} \pi^{-} \pi^{+}$ decay as an indication that both are $q \bar{q}$, or, perhaps $q q \bar{q} \bar{q}$ states. The evidence for the neutral $\kappa(800)$ would be endorsed if evidence for the charged $\kappa$ is also found. In the cases of both $\sigma$ and $\kappa$, a demonstration of the phase variation would be very welcome. It is also important to measure the $a_{0}(980)$ width in either $D_{s} \rightarrow K^{+} K^{-} \pi$ or $D_{s} \rightarrow \bar{K}^{0} K^{+} K^{-}$decays.

In the region above $1 \mathrm{GeV}$ more data is necessary to show which of the $f_{0}$ 's are genuine $q \bar{q}$ states. Perhaps the answer is none of those observed in scattering experiments, since the mixing between the bare $q \bar{q}$ resonances and the scalar glueball would not occur in charm decays. Apparently only one state appears in charm decays, although it is not clear yet which state this is. In any case, this state has no significant coupling to $K \bar{K}$.

There are important conceptual issues to be addressed. The most important is to formulate the unitarity constraint in multi-body decays. The assumption of two-body elastic scattering as the basic process is not trivial and may not be justified. Even in the case of two-body elastic scattering, the introduction of a relative phase can restore unitarity, which would be violated in models in which the amplitude is written as a sum of Breit-Wigners[11].

Decays of charm mesons, with their unique features, offer a new way to look at the light scalar mesons.

\section{References}

[1] F.Close and N. Törnqvist, hep-ph/0204205; S.Godfrey and J. Napolitano, Rev. Mod. Phys.71, 1411 (1999).

[2] E.M. Aitala et al, Phys. Rev. Lett. 86, 765 (2001).

[3] E.M. Aitala et al, Phys. Rev. Lett. 86, 770 (2001). 
[4] A. Bertin et al, Phys. Rev. D57, 55 (1998).

[5] C.Amsler and F.E.Close, Phys. Lett. B353, 385 (1995).

[6] H.Muramatsu et al, hep-ex/0207067.

[7] N.Wu et al, hep-ex/0104050.

[8] E.M. Aitala et al, Phys. Rev. Lett. 89, 121801 (2002).
[9] B.Aubert et al, hep-ex/0207089.

[10] P.L.Frabetti et al, Phys. Lett. B407, 79 (1997).

[11] M.Svec, Phys. Rev. D64:096003 (2001). 